\title{
FACT - Time-Resolved Blazar SEDs
}

\author{
D. Dorner*1, J. Adam ${ }^{2}$, M.L. Ahnen ${ }^{3}$, D. Baack ${ }^{2}$, M. Balbo ${ }^{4}$, A. Biland ${ }^{3}$, M. Blank ${ }^{1}$, \\ T. Bretz $^{3}{ }^{a}$, K. Bruegge ${ }^{2}$, M. Bulinski ${ }^{2}$, J. Buss ${ }^{2}$, A. Dmytriiev ${ }^{4}$, S. Einecke ${ }^{2}$, \\ D. Elsaesser ${ }^{2}$, C. Hempfling ${ }^{1}$, T. Herbst ${ }^{1}$, D. Hildebrand ${ }^{3}$, L. Kortmann ${ }^{2}$, \\ L. Linhoff ${ }^{2}$, M. Mahlke ${ }^{3} a$, K. Mannheim ${ }^{1}$, S.A. Mueller ${ }^{3}$, D. Neise ${ }^{3}$, A. Neronov ${ }^{4}$, \\ M. Noethe ${ }^{2}$, J. Oberkirch ${ }^{2}$, A. Paravac ${ }^{1}$, F. Pauss ${ }^{3}$, W. Rhode ${ }^{2}$, B. Schleicher ${ }^{1}$, \\ F. Schulz ${ }^{2}$, A. Shukla ${ }^{1}$, V. Sliusar ${ }^{4}$, F. Temme ${ }^{2}$, J. Thaele ${ }^{2}$, R. Walter ${ }^{4}$ (FACT \\ Collaboration), A. Kreikenbohm ${ }^{1}$, K. Leiter ${ }^{1}$ \\ ${ }^{1}$ Universität Würzburg, Institute for Theoretical Physics and Astrophysics, Emil-Fischer-Str. 31, \\ 97074 Würzburg, Germany \\ ${ }^{2}$ TU Dortmund, Experimental Physics 5, Otto-Hahn-Str. 4, 44221 Dortmund, Germany \\ ${ }^{3}$ ETH Zurich, Institute for Particle Physics, Otto-Stern-Weg 5, 8093 Zurich, Switzerland \\ ${ }^{4}$ University of Geneva, ISDC Data Center for Astrophysics, Chemin d'Ecogia 16, 1290 Versoix, \\ Switzerland \\ a also at RWTH Aachen University \\ E-mail: dornereastro.uni-wuerzburg.de
}

\begin{abstract}
Blazars are highly variable objects and their spectral energy distribution (SED) features two peaks. The emission at low energies is understood, however, the origin of the emission at $\mathrm{TeV}$ energies is strongly debated. While snapshots of SEDs usually can be explained with simple models, the evolution of SEDs challenges many models and allows for conclusions on the emission mechanisms. Leptonic models expect a correlation between the two peaks, while hadronic models can accommodate more complex correlations.

To study time-resolved SEDs, we set up a target-of-opportunity program triggering highresolution X-ray observations based on the monitoring at $\mathrm{TeV}$ energies by the First G-APD Cherenkov Telescope (FACT). To search for time lags and identify orphan flares, this is accompanied by X-ray monitoring with the Swift satellite. These observations provide an excellent multi-wavelength (MWL) data sample showing the temporal behaviour of the blazar emission along the electromagnetic spectrum.

To constrain the origin of the $\mathrm{TeV}$ emission, we extract the temporal evolution of the low energy peak from Swift data and calculate the expected flux at TeV energies using a theoretical model. Comparing this to the flux measured by FACT, we want to conclude on the underlying physics.

Results from more than five years of monitoring will be discussed.
\end{abstract}

35th International Cosmic Ray Conference - ICRC2017

10-20 July, 2017

Bexco, Busan, Korea

${ }^{*}$ Speaker. 


\section{FACT - Monitoring at TeV Energies}

The First G-APD Cherenkov Telescope (FACT) is operational since October 2011 in the Observatorio del Roque de los Muchachos at $2000 \mathrm{~m}$ a.s.l. It pioneered the usage of silicon based photosensors (SiPMs, aka Geiger-Mode Avalanche Photo Diodes, G-APDs) in ground-based gammaray astronomy. The excellent performance of SiPMs is highlighted in [1] and the performance of the telescope is discussed in [2]. As discussed in [3], SiPMs are ideally suited for the long-term monitoring and the observation strategy of the FACT project provides an unprecedented, unbiased data sample of blazars at $\mathrm{TeV}$ energies.

\section{FACT Analysis}

Within the FACT project, three analysis chains have been set up. Using Fact-Tools, the data are analyzed using modern data mining methods [4]. Based on the Modular Analysis and Reconstruction Software (MARS) package [5], the following two analysis chains have been set up.

\subsection{Quick Look Analysis}

To allow for low-latency alerts to the community in case of interesting events, a quick look analysis (QLA) was set up on site. The details of this analysis are discussed in [6]. The results of the QLA are provided publicly available on a website [7].

\subsection{Off-Site Analysis}

For off-site processing in the data center at the ISDC data center for astrophysics, new methods have been implemented and investigated.

Image Cleaning To go to lower energies, a new image cleaning algorithm was developed. To include fainter pixels, as a first step, islands with a maximum time difference of $17.5 \mathrm{~ns}$ are built, then neighbouring islands are merged and on the resulting image a size cut of 25 pe is applied. Monte Carlo studies show that more events with low energies survive the image cleaning.

Disp To calculate the disp, for the parametrization from [6] new coefficients have been optimized.

Background Suppression Similar to the background suppression cuts discussed in [6], a cut in Area versus Size is applied: Area $>\log 10($ Size $) * 898-1535$, where Area is the geometrical area of the shower image and Size is its photon content. In addition, a cut $\vartheta<0.156^{\circ}$ is applied.

\subsection{Data Selection}

Thanks to the stability of the SiPMs, the cosmic ray (CR) data themselves can be used to assess the data quality [8]. While a data quality selection based on the CR rate provides a very general way to study the data quality and is used to study the periodicity of the observed blazars [9], for the here presented analysis a data quality selection based on the rate after background suppression has been used adapting the method discussed in [10] for the complete time range of the QLA. 


\subsection{From Excess Rates to Fluxes}

While the QLA provides excess rates, fluxes are needed to compare the results with other instruments and combine data with different performance of the telescope (e.g. damaged mirror) or analysis (e.g. different background suppression). Calculating fluxes using Monte Carlo simulations as described in [2] requires a huge amount of simulations taking into account different zenith distances and night sky background conditions. To facilitate a quick estimate of the fluxes for the QLA, the excess rates of the Crab Nebula have been studied for periods of constant performance of the whole system. The effects of zenith distance and trigger threshold have been studied as described in [9] correcting the excess rates for these effects. Conversion factors from excess rate to Crab Units (CU) and fluxes using the measured spectrum of the Crab Nebula have been calculated.

\section{Flare Alerts from FACT}

Based on the QLA, a flare alert system has been set up accommodating various types of alerts as discussed in section 2.1 of [3]. While the QLA is available since December 2012 and the results publicly available since September 2013, official alerts are sent since March 2014. In total, already 44 alerts have been sent of which 30 were issued on 1ES 1959+650 with the majority during the exceptional flare in 2016.

\subsection{Mrk 501}

Mrk 501 Flare in June 2012 Already before 2014, interesting events were communicated. E.g. in May 2012, enhanced flux detected by FACT triggered detailed observations by MAGIC. A comparison of the FACT and MAGIC results has been shown in [11] and a detailed MWL study is being published [12].

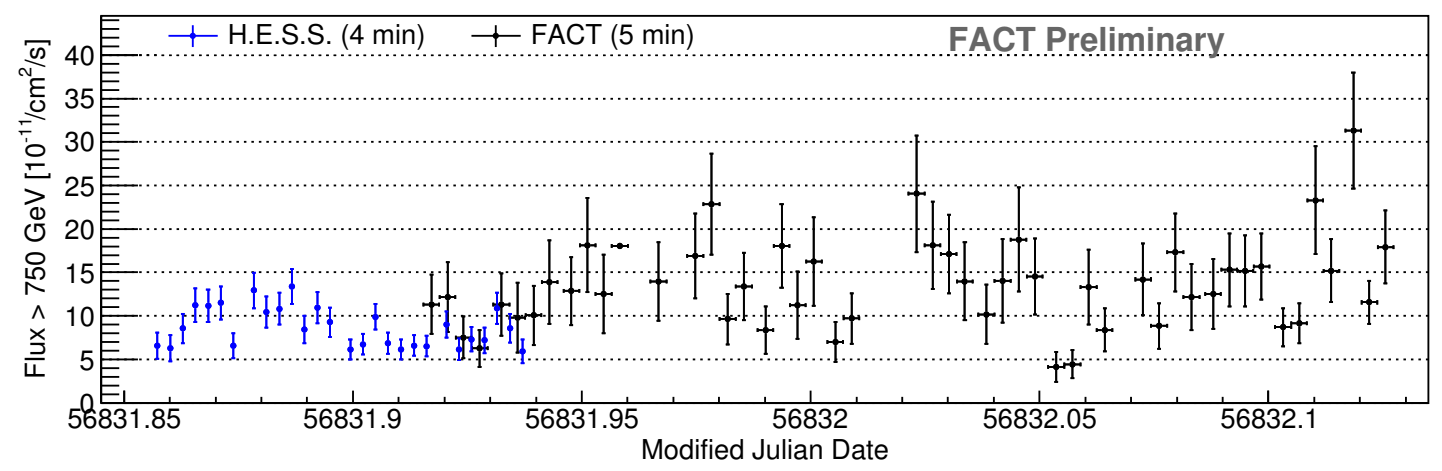

Figure 1: Light curve of the flare night combining measurements by H.E.S.S. (black, 4 min binning, from [13]) and FACT (red, 5 min binning).

Bright Flare of Mrk 501 in 2014 In June 2014, several alerts for Mrk 501 have been sent and H.E.S.S. carried out follow-up observations. Both FACT and H.E.S.S. observed the bright flare in the night of June 23./24. [14]. In Fig. 1, the intra-night light curve of the night with the brightest flux is shown. The integral fluxes by H.E.S.S. $(>2 \mathrm{TeV})$ have been converted to the different energy threshold by using the Mrk 501 spectrum measured during the flare night by H.E.S.S. [13]. A more detailed study including data from both instruments and more MWL data is ongoing [13]. 
The long-term light curves of Mrk 421 and Mrk 501 are discussed in [3].

\subsection{ES 1959+650}
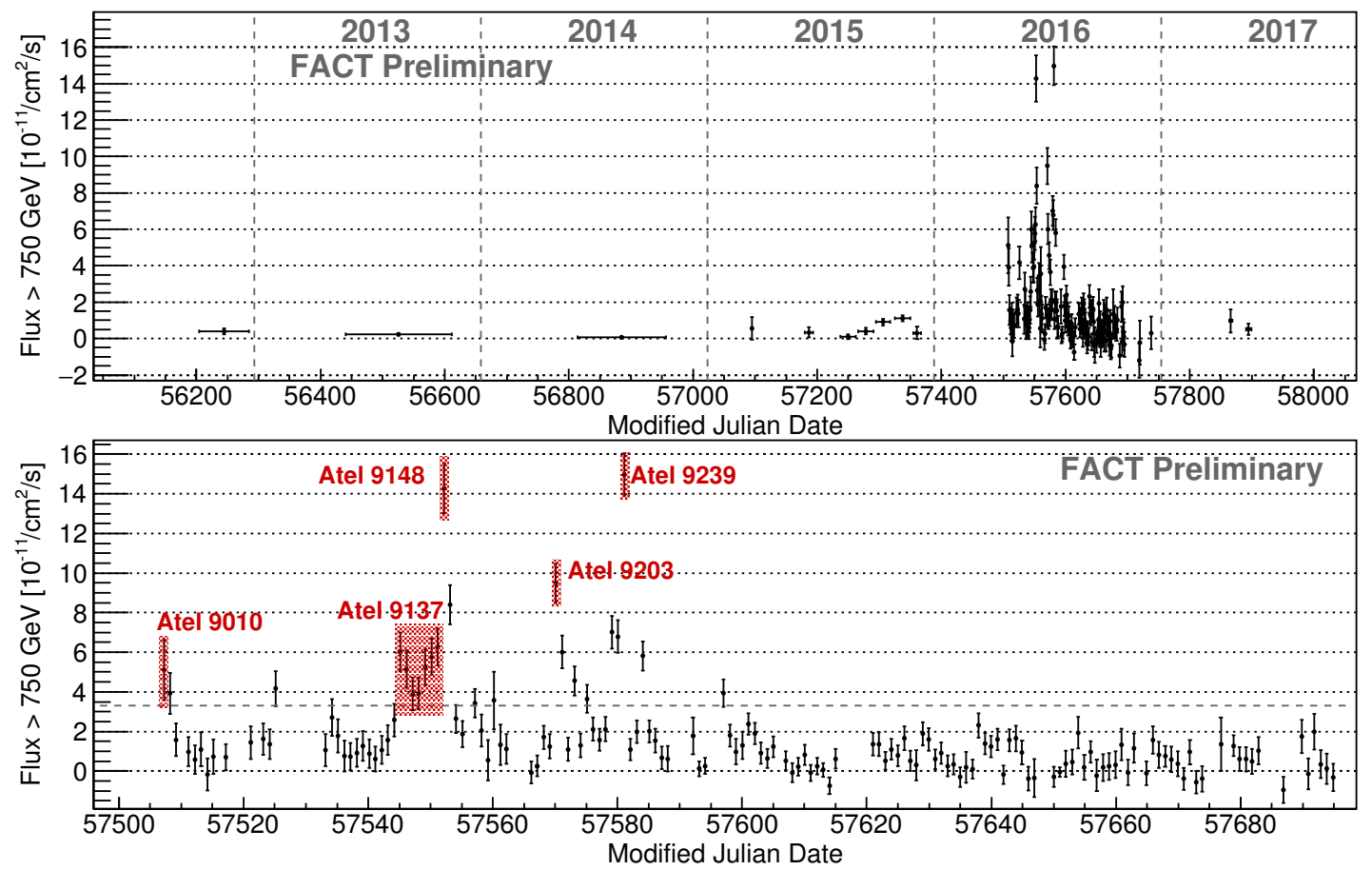

Figure 2: Light curve of 1ES 1959+650 as measured by FACT. In the top panel, the data are binned yearly for 2012-2014, monthly for 2015 and 2017, and nightly for 2016, according to the flux state of the source. The bottom panel shows a zoom to the light curve of 2016. Astromers's telegrams [15, 16, 17, 18, 19] are marked in red. The integral flux above $750 \mathrm{GeV}$ of the Crab Nebula is shown as dashed line.

Long-Term Behaviour Being a candidate for neutrino emission [20], 1ES 1959+650 has been monitored by FACT since 2012. While in the first years, the source was hardly detected, it started to show moderate activity in 2015 (see monthly bins, top panel, Fig. 2). In summer 2016, exceptional outbursts were recorded. After that, the source went back to the flux level of 2015.

Exceptional Flare of 1ES 1959+650 In 2016, the source had already moderate high flux level, when the observations were started [15]. A flux increase was measured [16] before the first bright flare in June [17]. In July, another bright flare [19] and some more activity [18] were observed. The FACT light curve of 2016 (bottom panel, Fig. 2) represents the densest daily light curve measured so far by an IACT.

\subsection{Target-of-Opportunity Program}

To study the variability processes, e.g. cooling, relevant for bright flares, a target-of-opportunity program has been set up and is active since 2013 . 
Program The base of the program is the unbiased FACT monitoring. In case a bright flare of more than $3 \mathrm{CU}$ is found, an immediate trigger is sent to X-ray satellite like e.g. INTEGRAL or XMM-Newton to measure time-resolved X-ray spectra of the flare and flux decay. To check for time-lags or orphan flares, the observations are accompanied with X-ray monitoring by Swift. The program was started with Mrk 421 and Mrk 501 and after its exceptional flare in 2016 also 1ES 1959+650 was included.

Trigger on Mrk 421 in December 2015 At the end of the visibility window for the X-ray satellites, a trigger on Mrk 421 was issued on December 16 in 2015. As the flare was shory and only of moderate flux, the flux had already decayed once the INTEGRAL observations were started. However, due to the X-ray monitoring and additional triggers to Swift, a dense MWL data sample was collected. Furthermore in June 2016 another outburst was recorded, which has also been seen by HAWC [21].

\section{Time-Resolved SEDs}

Studying blazars with spectral timing, different approaches are possible. Simultaneous SEDs can be compiled and fitted using different models. Interpreting the temporal evolution of the model parameters, models can be constrained. This approach is pursued with the target-of-opportunity program discussed above. However thanks to the flare alerts and the X-ray monitoring part of the program, a large MWL data sample is available providing the possibility for a different approach as described in the following.

\subsection{Predicting High Energy Light Curves}

As shown in Fig. 3, the gamma-ray flux is predicted assuming a homogeneous leptonic single zone Synchrotron Self-Compton (SSC) scenario within a canonical shock-in-jet model. The observed X-ray spectra from Swift-XRT (0.2-10 keV) were fit with the SSC model.

Model The emission is assumed to originate from a spherical region of radius $\mathrm{R}$ traveling along the jet. The region is moving relativistically and has a Doppler factor $\delta$. This relativistically moving region is filled with a random magnetic field of strength B and populated with relativistic electrons. The energy distribution of the electrons is assumed to be a broken power law distribution. The electron spectrum and magnetic field are tied up assuming that the origin of break in electron spectrum is the result of a classical synchrotron cooling break for a uniform emission region, $\Delta P=$ 1 and $\gamma_{c} \propto 1 / B^{2}$.

Method A $\chi^{2}$ minimization fit was performed to find the best-fit SED model to the observed Xray spectra. Based on this, integral $\gamma$-ray fluxes are computed above $700 \mathrm{GeV}$ by fitting X-ray data to the SSC model developed by [22]. This code was incorporated into the XSPEC spectral fitting software [23] as an external model to perform the minimization using the Levenburg-Marquadt algorithm. 


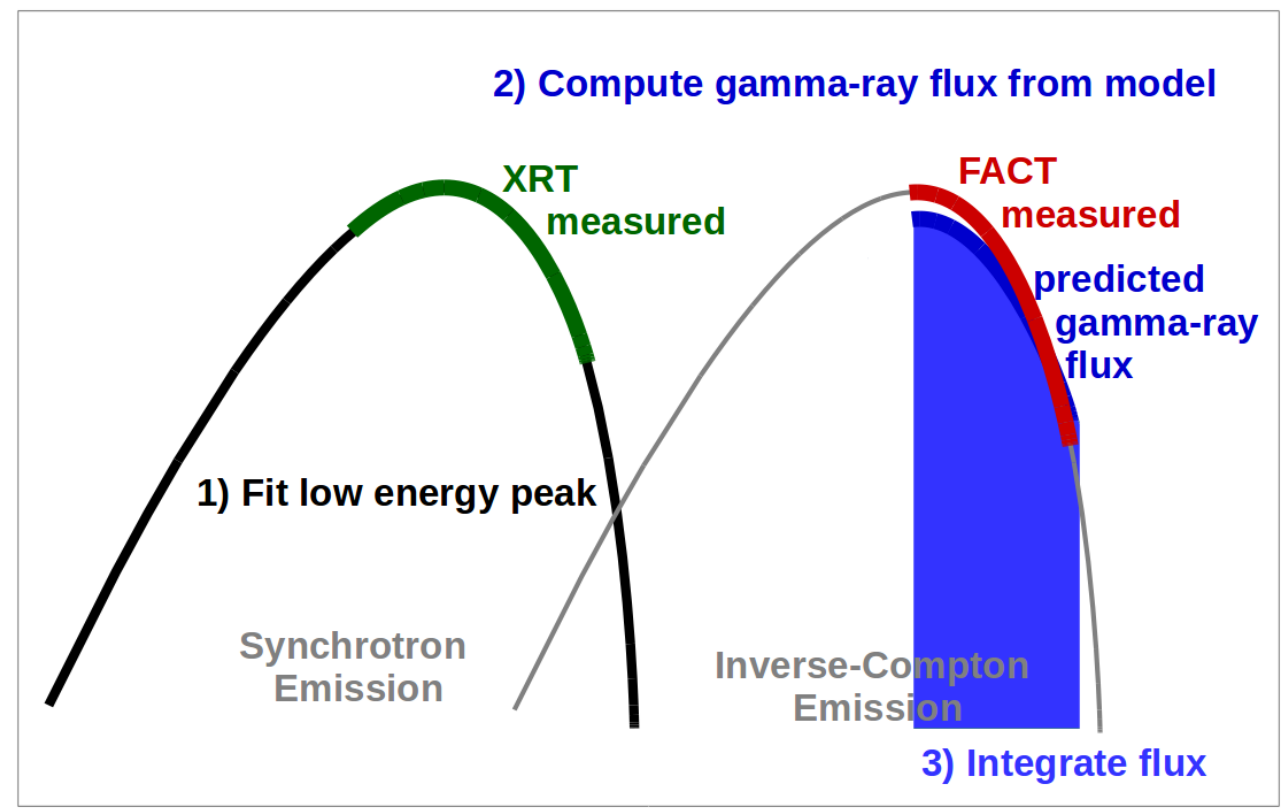

Figure 3: Schematics illustrating the method: Assuming a SSC model, the gamma-ray flux is predicted.

\subsection{Results}

A predicted light curve at $\mathrm{TeV}$ energies is calculated assuming a canonical shock-in-jet model scenario from Swift-XRT spectra. Currently, the study focusses on the first bright flare, as starting from MJD 57560 the X-ray data have less coverage and are less simultaneous. While moderate and low flux states are reproduced, for the brightest flare, which was observed by FACT on 12 June 2017, the flux is not predicted correctly see Fig 4. A possible explanation is that the bright flares originate from a different component which is not significantly contributing in the Swift-XRT energy band at $0.2-10 \mathrm{keV}$.

\section{Acknowledgments}

The important contributions from ETH Zurich grants ETH-10.08-2 and ETH-27.12-1 as well as the funding by the Swiss SNF and the German BMBF (Verbundforschung Astro- und Astroteilchenphysik) and HAP (Helmoltz Alliance for Astroparticle Physics) are gratefully acknowledged. Part of this work is supported by Deutsche Forschungsgemeinschaft (DFG) within the Collaborative Research Center SFB 876 "Providing Information by Resource-Constrained Analysis", project C3. We are thankful for the very valuable contributions from E. Lorenz, D. Renker and G. Viertel during the early phase of the project. We thank the Instituto de Astrofisica de Canarias for allowing us to operate the telescope at the Observatorio del Roque de los Muchachos in La Palma, the Max-Planck-Institut für Physik for providing us with the mount of the former HEGRA CT3 telescope, and the MAGIC collaboration for their support. 


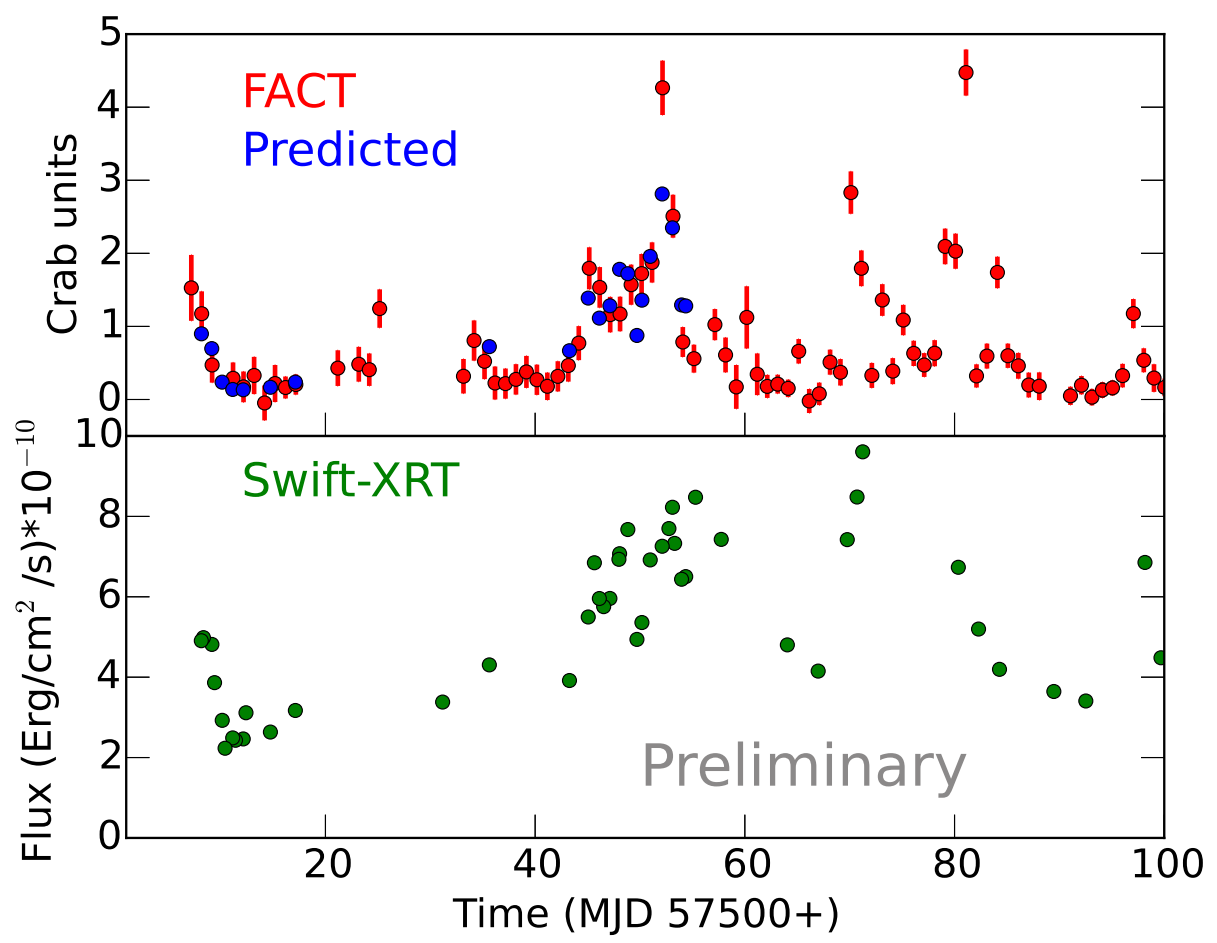

Figure 4: From the Swift-XRT spectra (light curve in bottom panel), the low energy peak has been fitted, predicting the high energy peak. Integrating the gamma-ray flux, the flux at $\mathrm{TeV}$ energies is predicted (blue points in upper panel) and compared with the measured flux from FACT (red points in upper panel). After MJD 57560 the X-ray data show less coverage and are less simultaneous.

\section{References}

[1] FACT collaboration, A. Biland, T. Bretz, J. Buß, V. Commichau, L. Djambazov, D. Dorner et al., Calibration and performance of the photon sensor response of FACT - the first G-APD Cherenkov telescope, Journal of Instrumentation 9 (Oct., 2014) P10012, [1403.5747].

[2] FACT collaboration, M. Noethe et al., FACT-Performance of the First Cherenkov Telescope Observing with SiPM, PoS ICRC2017 (2017) 791.

[3] FACT collaboration, D. Dorner et al., FACT - Highlights from more than Five Years of Unbiased Monitoring at TeV Energies, PoS ICRC2017 (2017) 609.

[4] FACT collaboration, K. A. Brügge et al., FACT-Tools: Streamed Real-Time Data Analysis, in 34th International Cosmic Ray Conference (ICRC2015), vol. 34 of International Cosmic Ray Conference, p. 865, July, 2015.

[5] T. Bretz and D. Dorner, MARS - CheObs ed. - A flexible Software Framework for future Cherenkov Telescopes, in Astroparticle, Particle and Space Physics, Detectors and Medical Physics Applications (C. Leroy, P.-G. Rancoita, M. Barone, A. Gaddi, L. Price and R. Ruchti, eds.), pp. 681-687, Apr., 2010, DOI.

[6] D. Dorner, M. L. Ahnen, M. Bergmann, A. Biland, M. Balbo, T. Bretz et al., FACT - Monitoring Blazars at Very High Energies, ArXiv e-prints (Feb., 2015), [1502. 02582]. 
[7] FACT collaboration, D. Dorner and et al., "FACT Quick-Look Analysis: http://www.fact-project.org/monitoring."

[8] FACT collaboration, D. Hildebrand et al., Using Charged Cosmic Ray Particles to Monitor the Data Quality of FAC, PoS ICRC2017 (2017) 779.

[9] FACT collaboration, M. Mahlke et al., FACT - Searching for periodicity in five-year light-curves of Active Galactic Nuclei, PoS ICRC2017 (2017) 612.

[10] D. Dorner, A. Biland, T. Bretz, J. Buss, S. Einecke, D. Eisenacher et al., FACT - Long-term Monitoring of Bright TeV-Blazars, ArXiv e-prints (Nov., 2013) , [1311. 0478 ].

[11] FACT collaboration, D. Dorner et al., FACT - TeV Flare Alerts Triggering Multi-Wavelength Observations, in 34th International Cosmic Ray Conference (ICRC2015), vol. 34 of International Cosmic Ray Conference, p. 704, July, 2015.

[12] FACT, MAGIC, VERITAS collaboration, M. Ahnen and et al., The extreme HBL behavior of Mrk 501 during 2012, in prep. (2017).

[13] FACT, H.E.S.S. collaboration, G. Cologna, N. Chakraborty, A. Jacholkowska, M. Lorentz, M. Mohamed, C. Perennes et al., The Exceptional Flare of Mrk 501 in 2014: Combined Observations with H.E.S.S. and FACT, ArXiv e-prints (Nov., 2016) , [1611.03983].

[14] H.E.S.S. collaboration, C. Stegmann, Increased VHE activity from Mrk 501 detected with H.E.S.S., The Astronomer's Telegram 6268 (June, 2014) .

[15] FACT, FERMI, MAGIC, VERITAS collaboration, S. Buson, J. D. Magill, D. Dorner, A. Biland, R. Mirzoyan and R. Mukherjee, Fermi-LAT, FACT, MAGIC and VERITAS detection of increasing gamma-ray activity from the high-energy peaked BL Lac object 1ES 1959+650, The Astronomer's Telegram 9010 (Apr., 2016) .

[16] FACT collaboration, A. Biland, FACT measures increased gamma-ray flux from the high-energy peaked BL Lac object 1ES 1959+650 since five nights, The Astronomer's Telegram 9139 (June, 2016)

[17] FACT, FERmi, MAGIC, VERITAS collaboration, A. Biland, D. Dorner, R. Mirzoyan, R. Mukherjee, S. Buson and B. Kapanazde, Further increase of gamma-ray emission from the HBL IES 1959+650, The Astronomer's Telegram 9148 (June, 2016) .

[18] FACT, MAGIC collaboration, A. Biland and R. Mirzoyan, FACT and MAGIC measure an increased gamma-ray flux from the HBL 1ES 1959+650, The Astronomer's Telegram 9203 (July, 2016) .

[19] FACT collaboration, A. Biland, FACT measures new maximum flux from the HBL 1ES 1959+650 at TeV energies, The Astronomer's Telegram 9239 (July, 2016) .

[20] F. Halzen and D. Hooper, High energy neutrinos from the TeV Blazar 1ES 1959 + 650, Astroparticle Physics 23 (July, 2005) 537-542, [astro-ph / 0502449 ].

[21] FACT AND HAWC collaboration, A. Biland et al., Enhanced and increasing activity in gamma rays and X-rays from the HBL Mrk421, ATel 9137 (2016) .

[22] H. Krawczynski, S. B. Hughes, D. Horan, F. Aharonian, M. F. Aller, H. Aller et al., Multiwavelength Observations of Strong Flares from the TeV Blazar 1ES 1959+650, ApJ 601 (Jan., 2004) 151-164, [astro-ph/0310158].

[23] K. A. Arnaud, XSPEC: The First Ten Years, in Astronomical Data Analysis Software and Systems V (G. H. Jacoby and J. Barnes, eds.), vol. 101 of Astronomical Society of the Pacific Conference Series, p. 17, 1996. 